\title{
Structural Phase Transition and Electronic Properties of NdBi
}

\author{
Ashvini K Sahu ${ }^{1,2^{*}}$, R Bhardwaj ${ }^{2}$, A P Jain ${ }^{2}$, Sankar P Sanyal ${ }^{1}$ \\ ${ }^{1}$ Department of Physics, Barkatullah University, Bhopal 462 026, India \\ ${ }^{2}$ Truba Institute of Engineering \& Information Technology, Bhopal 462 023, India \\ * E-mail of corresponding author: asavanisahu@gmail.com
}

\begin{abstract}
The structural and electronic properties of NdBi from an electronic structure calculation have been presented. The calculation is performed using self-consistent tight binding linear muffin tin orbital (TB-LMTO) method within the local density approximation (LDA). The calculated equilibrium structural parameters are in good agreement with the available experimental results. It is found that this compound shows metallic behavior under ambient condition and undergoes a structural phase transition from the $\mathrm{NaCl}$ structure to the $\mathrm{CsCl}$ structure at the pressure $20.1 \mathrm{GPa}$. The electronic structures of NdBi under pressure are investigated. It is found that $\mathrm{NdBi}$ have metalliation and the hybridizations of atoms in NdBi under pressure become stronger.
\end{abstract}

Keywords: Phase transition, bulk modulus, band structure, density of states.

\section{Introduction}

The mono-pnictides of rare earth with $\mathrm{NaCl}$ structure in recent years have drawn a great attention because of their diversive and unusual properties. The structural properties of several pnictides of lanthanide group, which crystallize in $\mathrm{NaCl}$ type crystal structure have been investigated by using high-pressure X-ray diffraction technique [1-5] and reported to undergo to either $\mathrm{CsCl}$ or body centered tetragonal (BCT) structure. The X-ray diffraction of cerium mono pnictides has been studied up to $30 \mathrm{GPa}$ at room temperature [5-6]. Amongst all the Ce pnictides, only $\mathrm{CeP}$ exhibits an isomorphous transition about $10 \mathrm{GPa}$, which is associated to an electronic transition involving the change in the valence state of $\mathrm{Ce}$ [6]. Furthermore this compound transforms from $\mathrm{NaCl}$ structure to $\mathrm{CsCl}$ phase around $19 \mathrm{GPa}$. The high-pressure phases of other pnictides of Ce are either BCT or $\mathrm{CsCl}$. The high-pressure forms of the bismuthides of $\mathrm{Ce}$ and $\mathrm{Pr}$ are reported as the co-existing cubic $\mathrm{CsCl}$ and tetragonal phases [7]. Gd mono pnictides $(\mathrm{GdX}, \mathrm{X}=\mathrm{N}, \mathrm{P}, \mathrm{As}, \mathrm{Sb}, \mathrm{Bi})$ are known to exhibit unusual electric and magnetic $[8,9]$ phenomena. By use of synchrotron radiation, powder X-ray diffraction studied for $\mathrm{LnBi}(\mathrm{Ln}=\mathrm{La}$ and $\mathrm{Pr}$ ) with the $\mathrm{NaCl}$-type structure have been carried out up to $30 \mathrm{GPa}$ at room temperature. The structural phase transition of $\mathrm{LaBi}$ occurs at about $11.5 \mathrm{GPa}$. The high-pressure phase of LaBi consists of coexisting of tetragonal $(\mathrm{P} 4 / \mathrm{mmm})$ and cubic $(\mathrm{Pm} 3 \mathrm{~m})$ and cubic $(\mathrm{Pm} 3 \mathrm{~m})$ modifications at about $14 \mathrm{GPa}$ [10]. Hayashi et al. [11] using synchrotron radiation have studied the X-ray diffraction of NdBi with the NaCl-type structure up to $27 \mathrm{GPa}$ at room temperature.

\section{Method of Calculation}

The total energy, BS and DOS for NdBi are calculated using a similar procedure as reported in our pervious work [12-14] using the TB-LMTO method [15, 16] within the local density approximation (LDA) [17]. Van Barth and Hedin [18] parameterization scheme has been used for exchange correlation potential. The NdBi compound crystallize in the NaCl-type structure ( $f c c$ lattice with space group, $F m 3 m$, No. 225). In the $\mathrm{NaCl}$ structure, the $\mathrm{Nd}$ and $\mathrm{Bi}$ atoms are located at the position: $\mathrm{Nd}:(0,0.0)$ and $\mathrm{Bi}:(0.5,0.5,0.5)$. The structure of the high-pressure phase is CsCl-type ( $B_{2}$-phase) ( $s c$ lattice with space group, $P m 3 m$, No. 221) with positions of $\mathrm{Nd}:(0,0,0)$ and $\mathrm{Bi}:(0.5,0.5,0.5)$. As mention earlier work $[12,14,19]$, the TB-LMTO method works well for the close-packed structure and since the $\mathrm{NdBi}$ compounds belongs to NaCl-type ( $B_{1}$-phase) structure at ambient conditions, which is not a close-packed one. We have therefore introduced two equivalent empty spheres at position $(0.25,0.25,0.25)$ and $(0.75,0.75,0.75)$ in such a way that they do not break the crystal symmetry [20]. However, the CsCl-type structure ( $B_{2}$-phase) is a closed-packed structure, and no such empty spheres were needed. The Wigner-Seitz sphere was chosen in such a way that the sphere boundary potential is minimum and the charge flow between the atoms is in accordance with the electro negativity criteria [14, 19]. The E and $\boldsymbol{k}$ convergences are checked subsequently to achieve better accuracy. The calculations were performed for $512 \boldsymbol{k}$ points (grid of $8 \times 8 \times 8$ ) in the Brillouin zone for both the $B_{1}$ and $B_{2}$ phases. The tetrahedron method [21] of Brillouin zone integration has been used to calculate the DOS. The total energy was computed by reducing the volume from $1.05 V_{0}$ to $0.60 V_{0}$, where $V_{0}$ is the equilibrium cell volume. The calculated total energy was fitted to Birch equation of sates [22] to obtain the pressure volume relation. The pressure is obtained by taking the volume derivative of total energy. The Bulk modulus $B_{T}\left(-V_{0} d P / d V_{0}\right)$ is also calculated from the $P-V$ relations. The stability of a particular structure is decided by the minima of the enthalpy. The phase-transition pressure $P_{t}$ can be obtained by matching enthalpies of both structure such that the difference of enthalpy $\Delta H\left(=H_{B 2}-H_{B 1}\right)$ becomes zero at transition pressure $\left(P_{t}\right)$. 


\section{Results and discussion}

\subsection{Structural properties}

The electronic BS calculations are performed to estimate the total energy of NdBi compound by using the first principle TB-LMTO method. The total energies for NdBi compound are plotted against different compressions and are shown in Figure 1.

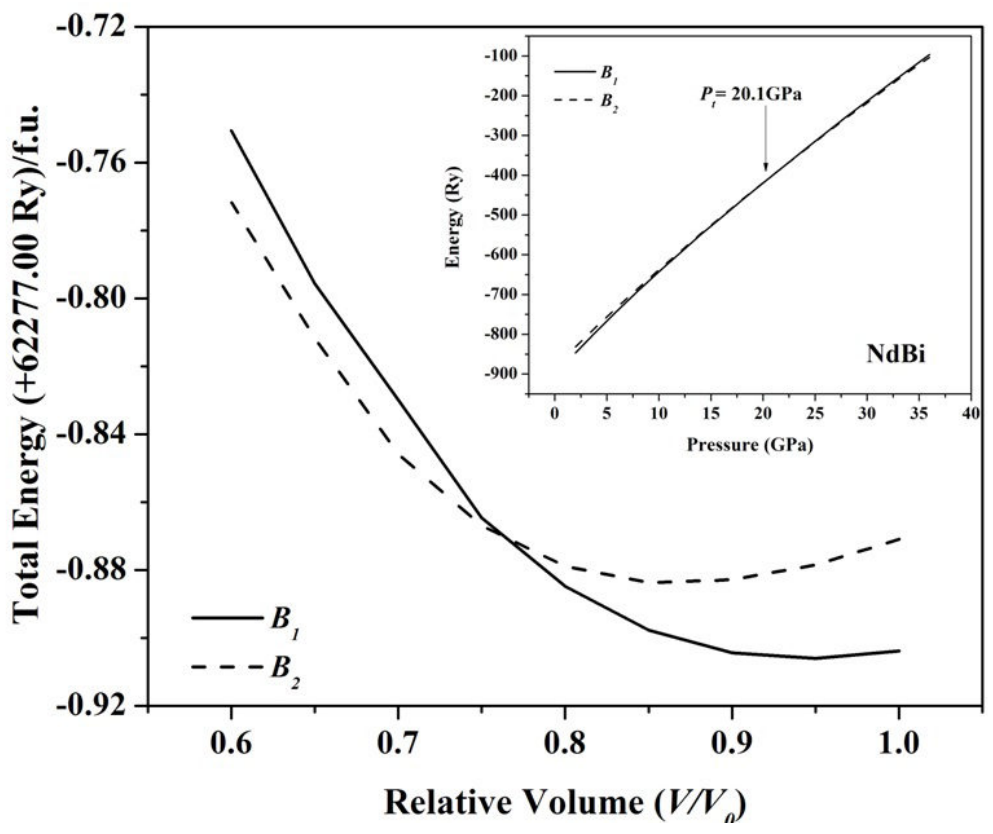

Figure 1.Variation of total energy with relative volume for the $B_{1}$ and $B_{2}$ phase for $\mathrm{NdBi}$. The inset shows the variation of enthalpies in the $B_{1}$ and $B_{2}$ phases for $\mathrm{NdBi}$.

The minimum of all the curves define the equilibrium volume $V_{0}$ (or equilibrium separation $a_{0}$ ), which is found to be $62.5 \AA^{3}$ and the corresponding lattice parameter is $6.3 \AA$, which is underestimated by $1 \%$ for NdBi as compared to the experimental data [11], which is listed in Table 1.

Table 1. Calculated equilibrium lattice parameter $a_{0}(\AA)$, bulk modulus $B_{T}(\mathrm{GPa})$, number of $f$-states at the Fermi level $\mathrm{N}\left(E_{f}\right)$ (states/Ry cell), phase transition pressure $P_{t}$ in $(\mathrm{GPa})$ of TM Nitrides in the $B_{l}$ and $B_{2}$ phases.

\begin{tabular}{|c|c|c|c|c|c|c|c|c|}
\hline \multirow[t]{2}{*}{ Solids } & & \multicolumn{2}{|c|}{$a_{0}=2 \mathrm{r}(\AA)$} & \multicolumn{2}{|c|}{$B_{T}(\mathrm{GPa})$} & \multirow{2}{*}{$\begin{array}{c}\mathrm{N}\left(E_{f}\right) \\
\text { (states/Ry cell) }\end{array}$} & \multirow{2}{*}{$\begin{array}{c}P_{t} \\
(\mathrm{GPa})\end{array}$} & \multirow{2}{*}{$\begin{array}{l}\left(V / V_{0}\right) \\
\%\end{array}$} \\
\hline & & $B_{1}$ & $B_{2}$ & $B_{1}$ & $B_{2}$ & & & \\
\hline $\mathrm{NdBi}$ & Pres. & 6.3 & 3.9 & 61.5 & 54.7 & 684.07 & 20.1 & 2 \\
\hline & Expt & $6.4^{\mathrm{a}}$ & -- & -- & -- & -- & $21.1^{\mathrm{a}}$ & -- \\
\hline
\end{tabular}

\footnotetext{
${ }^{a} \operatorname{Ref}[11]$
}

The present values of lattice parameters are in good agreement with the experimental values [11]. However, experimentally [11] reported values of the lattice parameter for $\mathrm{NdBi}$ is little larger than our results. This is primarily due to usage of LDA [17] in the present calculation. Regarding the LDA contraction, it is often found that the LDA leads to some over binding which yields lattice parameters that are somewhat smaller when compared with the experimental.

The calculation of transition pressure is carried out by estimating enthalpy in both the structures, as mentioned in Section 2. We have, therefore, plotted the variation of enthalpy with pressure for $\mathrm{NdBi}$ in the $B_{1}$ and $B_{2}$ phases, shown as inset of Figure 1.

The pressure at which these two phases coexist is phase-transition pressure. The equation of state in terms of pressure variation of relative volume change is given in Figure 2 for NdBi. 


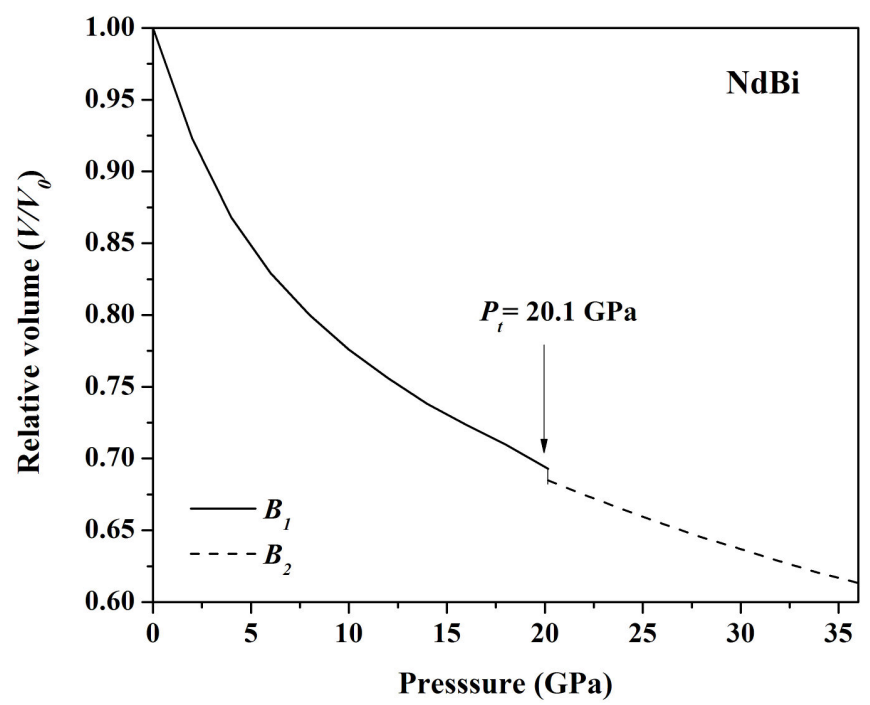

Figure 2. Equation of states for $\mathrm{NdBi}$ compound. Solid line represents the $B_{l}$ phase and dotted line represents the $B_{2}$ phase.

In the case of $\mathrm{NdBi}$, we have predicted a phase transition pressure of $20.1 \mathrm{GPa}$ with relative volume collapse of $2 \%$. The calculated values of phase transition pressure for $\mathrm{NdBi}$ is compared with experimental results reported so far [11]. The calculated bulk modulus value for $\mathrm{NdBi}$ is listed in Table 1.

\subsection{Electronic properties}

The electronic BS and the DOS for the NdBi in their $B_{l}$-phase under ambient conditions are shown in Figure 3. From these figures, it can be noticed that these compounds show metallic property which is manifested by the presence of large number of $d$ - and $f$-states at the Fermi level for NdBi respectively, as mentioned in Table 1.

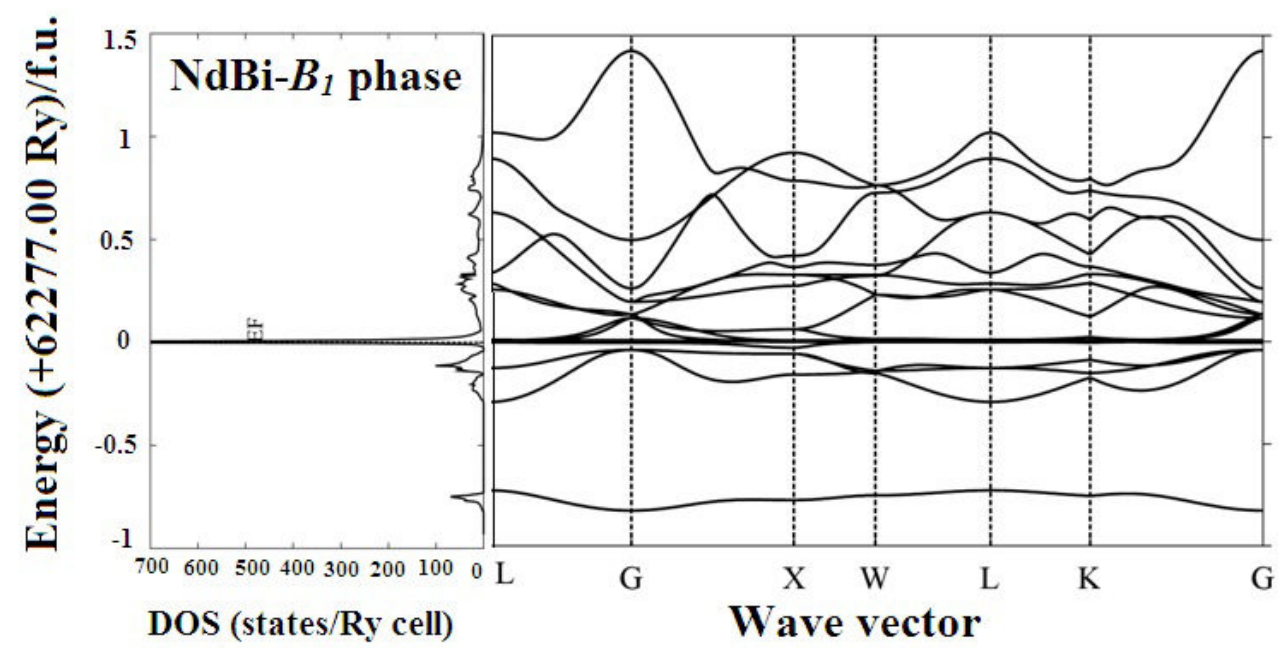

Figure $3 \mathrm{BS}$ and DOS in the $B_{l}$ phase for $\mathrm{NdBi}$.

To understand the elementary contribution of all atoms, we have studied the partial density of states (PDOS) which are shown in Figure 4. From the PDOS analysis, it has been found that the lower valence band lying around $-0.75 \mathrm{Ry}$ is due to the Bi-s states for NdBi. 

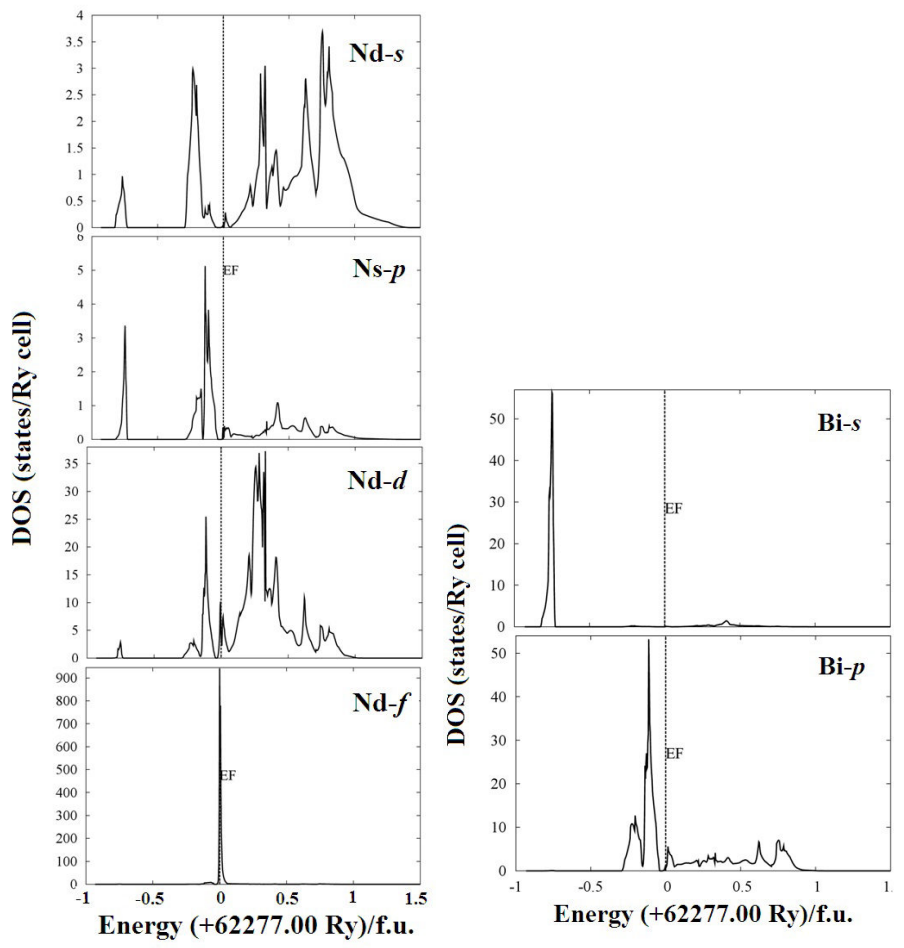

Figure 4. PDOS in the $B_{I}$ phase for NdBi.

The upper valence band lying between -0.5 and 0.0 Ry are due to hybridization of Nd-s, $p$-, $d$ - and Bi- $p$ states for $\mathrm{NdBi}$ The Nd- $f$ and Bi- $p$ states are highly localized at the Fermi level. The conduction bands are due to the hybridization of the Nd- $f$ and Bi- $p$ states at the Fermi level for NdBi. As pointed out earlier, the NdBi compound undergoes structural phase transition from the $B_{1}$ to $B_{2}$ phases at the pressure of $20.1 \mathrm{GPa}$. Thus, the electronic BS and DOS for both compounds in the $B_{2}$-phase are shown in Figure 5 .

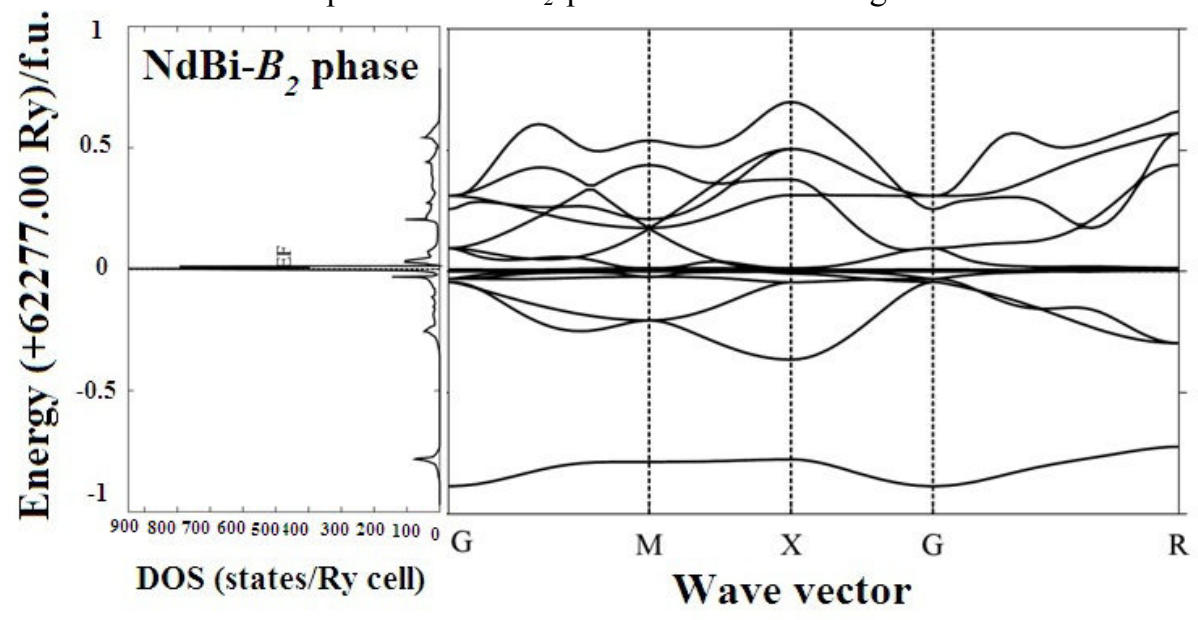

Figure 5. BS and DOS in the $B_{2}$ phase for NsBiTiN and $\mathrm{NbN}$ respectively.

The NdBi retain their metallic property at high pressure also. The nature of the bands and DOS in the $B_{2}$-phase is similar to the $B_{I}$-phases, with a minor difference that the lower energy bands are shifted to the higher energy bands near the Fermi level and more compressed bands are seen for NdBi. The hybridization of the Nd- $d,-f$ and Bi- $p$ states in the conduction band increases due to increase in pressure.

\section{Conclusions}

In summary, we have investigated structural and electronic properties for NdBi using the TB-LMTO method within the LDA. At ambient conditions, this compound is stable in the NaCl-type structure and undergoes structural phase transition to the CsCl-type structure in the pressure $20.1 \mathrm{GPa}$. The lattice parameters, bulk modulus and the phase transition pressure are obtained which are in good agreement with the experimental data. 
The electronic properties of $\mathrm{NdBi}$ under pressure are also calculated, and it is found that NdBi exhibit a metallic behavior and the hybridizations between atoms become stronger under pressure.

\section{Acknowledgements}

The authors are thankful to UGC-(SAP) for financial assistance. SPS is thankful to MPCST for partial financial support. RKB is thankful to MPCST for partial financial support.

\section{References}

[1] J. M. Leger, D. Ravot, J. Rossat Mignod, J. Phys. C 17 (1984) 4935.

[2] T. Adachi, I. Shirotani, J. Hayashi, O. Shimomura, Phys. Lett. A 250 (1998) 389.

[3] I. Shirotani, K. Yamanashi, J. Hayashi, Y. Tanaka, N. Ishimatsu, O. Shimomura, T. Kikegawa, J. Phys, Condens, Matter 13 (2001) 1939.

[4] I. Vedel, A. M. Redon, J. Rossat Mignod, O. Vogt, J. M. Leger, J. Phys. C: Solid State Phys. 20 (1987) 3439.

[5] A. Werner, H. D. Hocheimer, R. L. Meng E. Bucher, Phys. Lett. A 97 (1983) 207.

[6] A. Jayaraman, W. Lowe, L. D. Longinotti, E. Butcher, Phys. Rev. Lett. 36 (1976) 366.

[7] I. Shirotani, J. Hayashi, K. Yamanashi, K. Hirano, T. Adachi, N. Ishimatsu, O. Shimomura, T. Kikegawa, Physica B 334 (2003) 167.

[8] D. X. Li, Y. Haga, H. Shida, T. Suzuki, Y. S. Kwon and G. Kubo, J. Phys.: Condens. $\quad$ Matter 9 (1997) 10777.

[9] T. Tomimatsu, K. Koyama, M. Yoshida, D. Li and M. Motokawa Phys. Rev. B 67 (2003) 014406.

[10] J. Hayashi, T. Toyama, N. Hoshi, I. Shirotani, T. Kikegawa, PF-activity report (2005), \#23, part B, (2006) 187.

[11] J. Hayashi, H. Ando, Y. Sugiuchi and I. Shirotani PF-activity report (2006) \#24, part B, 199.

[12] D. B. Singh, V. Srivastava, M. Rajagopalan, M. Husain, and A.K. Bandyopadhyay,Phys. Rev. B 64 (2001) 115110

[13] V. Srivastava, M. Rajagopalan, and S.P. Sanyal, Eur. Phys. J. B 61 (2008) 131.

[14] P. Pandit, V. Srivastava, M. Rajagopalan, and S.P. Sanyal, Physica B 403 (2008) 433.

[15] The source of the computer code can be downloaded with permission from Prof. Andersen 5Andersen@and.mpi-stuttgart.mpg.de4. However, documentation about the installation and run of the TB-LMTO code can be downloaded from the website: cst5www.nrl.navy.mil/users/mazin/LMTODOC.pdf4; O.K. Andersen, Phys. Rev. B. 12 (1975) 3060.

[16] O. K. Andersen and O. Jepsen, Phys. Rev. Lett. 53 (1984) 2571.

[17] W. Kohn and L. J. Sham, Phys. Rev. A 140 (1965) 1133.

[18] U. Van Barth and L. Hedin, J. Phys. C 5 (1972) 1629.

[19] V. Srivastava, M. Rajagopalan, and S.P. Sanyal, J. Magn. Magn. Mater 321 (2009), 607.

[20] N. E. Christensen, Phys. Rev. B 32 (1985) 207.

[21] O. Jepsen and O.K. Andersen, Solid State Commun. 9 (1971) 1763.

[22] F. Birch, J. Geophys. Rev. 83 (1978) 1257. 
This academic article was published by The International Institute for Science, Technology and Education (IISTE). The IISTE is a pioneer in the Open Access Publishing service based in the U.S. and Europe. The aim of the institute is Accelerating Global Knowledge Sharing.

More information about the publisher can be found in the IISTE's homepage: http://www.iiste.org

\section{CALL FOR PAPERS}

The IISTE is currently hosting more than 30 peer-reviewed academic journals and collaborating with academic institutions around the world. There's no deadline for submission. Prospective authors of IISTE journals can find the submission instruction on the following page: http://www.iiste.org/Journals/

The IISTE editorial team promises to the review and publish all the qualified submissions in a fast manner. All the journals articles are available online to the readers all over the world without financial, legal, or technical barriers other than those inseparable from gaining access to the internet itself. Printed version of the journals is also available upon request of readers and authors.

\section{IISTE Knowledge Sharing Partners}

EBSCO, Index Copernicus, Ulrich's Periodicals Directory, JournalTOCS, PKP Open Archives Harvester, Bielefeld Academic Search Engine, Elektronische Zeitschriftenbibliothek EZB, Open J-Gate, OCLC WorldCat, Universe Digtial Library, NewJour, Google Scholar

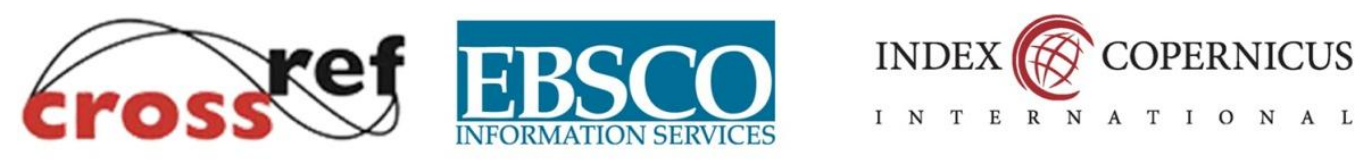

(O) ULRICHSWEB"

JournalTOCs

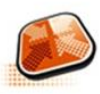

PKP | PUBLIC KNOWLEDGE PROJECT
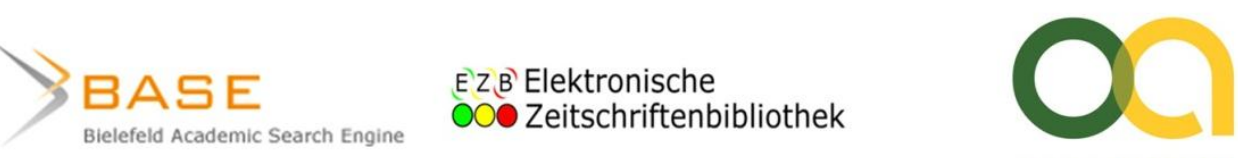

open access
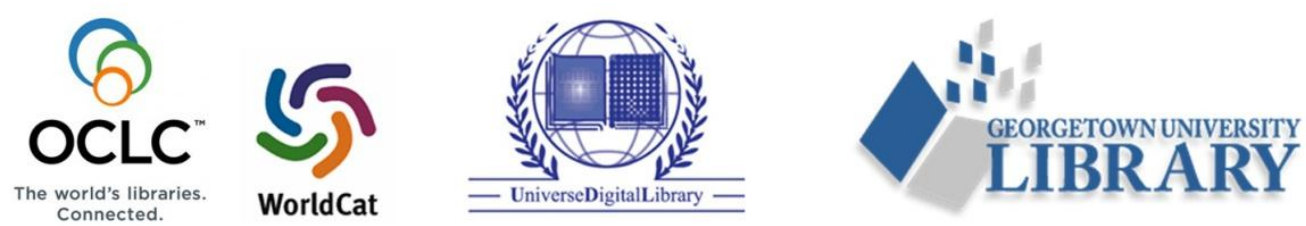